\title{
LAS OBRAS HIDRÁULICAS EN LOS SISTEMAS FLUVIALES \\ DE LA COSTA BRAVA: PREFERENCIAS LOCALES Y \\ LIMITACIONES DE UN MODELO CONVENCIONAL DE ADAPTACIÓN AL RIESGO DE INUNDACIÓN ${ }^{1}$
}

Dolors Roset Pagès², David Saurí Pujol ${ }^{3}$

y Anna Ribas Palom²

\section{RESUMEN}

Este artículo tiene por finalidad analizar las medidas de adaptación al riesgo de inundación que se han realizado en el ámbito de la Costa Brava, con especial incidencia en el papel que han tenido las obras de infraestructura hidráulica en la prevención de avenidas. Se intenta comprobar hasta que punto la percepción local dominante sobre las obras hidráulicas como una de las formas más eficientes para la prevención de inundaciones se contradice con las nuevas tendencias tanto en relación a los costes ecológicos de la construcción de estos dispositivos hidráulicos como al planeamiento urbanístico y fluvial, en general.

Palabras clave: riesgo, inundación, Costa Brava, obras hidráulicas.

RESUMÉ

Cet article analyse les mesures de gestion du risque d'inondation à la Costa Brava, en mettant en lumière le rôle important qu'ont joué les infrastructures hydrauliques dans la prévention des inondations. On essai de prouver jusqu à quel point la perception locale dominante sur les infrastructures hydrauliques comme une des formes les plus efficaces pour la prévention des inondations se contradie avec les nouvelles tendences tant en relation aux impacts écologiques de cettes infrastructures autant comme en relation à l'aménagement urbanistique et fluviale, en general.

Mots clés: risque, inondation, Costa Brava, infrastructures hydrauliques.

Este estudio forma parte del proyecto de investigación financiado por la CICYT «El riesgo de inundación en los núcleos turísticos de la Costa Brava. Balance actual y perspectivas futuras» (AMB93-0665).

2 Sección de Geografía de la Universitat de Girona.

3 Departamento de Geografía de la Universitat Autònoma de Barcelona. 
This article focuses on adaptative policies to the flood hazard in the area of the Costa Brava. A special emphasis is put on the role of hydraulic infrastructure in flood prevention. We attempt to examine the extent to which local perception of hydraulics works as the more efficient form to adaptation to these phenomena clashes with new flood control policies such as land use planning or river basin planning in general.

Key words: hazard, flood, Costa Brava, hydraulic infrastructure.

\section{Introducción}

Las medidas preventivas estructurales y de responsabilidad colectiva, como las obras hidráulicas, representan las formas de adaptación a las inundaciones fluviales que más confianza han generado entre las sociedades tanto tradicionales como modernas. No es de extrañar, por tanto, que la mayor parte de los cursos fluviales del litoral mediterráneo hayan sido modificados en mayor o menor grado a través de obras de acondicionamiento que van desde muros de contención, embalses de laminación, dragados del lecho del río, hasta llegar a actuaciones tan extremas como el desvío o incluso el total recubrimiento del curso fluvial. Sin embargo, la efectividad social de estas obras hidráulicas podría hallarse en entredicho desde el momento que los crecientes costes económicos, sociales y ambientales de estas obras, junto al hecho que tampoco garantizan una protección absoluta frente a las inundaciones, empiezan a ser tenidos en cuenta desde diversos colectivos de expertos en la ordenación y gestión de cursos fluviales (Calvo, 1984). A pesar de esta tendencia, la solución que según la Administración Local resuelve mejor la problemática de las inundaciones continua siendo la construcción de obras de infraestructura hidráulica.

El objetivo de este artículo es hacer una aproximación a la percepción social en relación a las obras hidráulicas como medida de adaptación a las inundaciones en el ámbito territorial de la Costa Brava. Los ríos y rieras litorales de la Costa Brava cuentan con buen número de obras de infraestructura hidráulica, pero paradójicamente los daños que causan las inundaciones son cada vez más importantes. Ello nos lleva a pensar que puede tratarse de un ejemplo claro de lo que viene denominándose «efecto escalada del riesgo»: cuando en un determinado espacio se consiguen teóricamente mayores niveles de seguridad frente a las inundaciones, el crecimiento urbanístico en espacios tradicionalmente inundables provoca que la protección frente a estos episodios sea progresivamente menor (Parker, 1995).

A fin de conocer las preferencias de las distintas administraciones competentes en la gestión de ríos y rieras de los municipios que conforman la Costa Brava en lo que se refiere a formas de adaptación a las inundaciones se llevaron a cabo entrevistas en profundidad (en total 21) a responsables municipales de los ayuntamientos de la Costa Brava (Portbou, Colera, Llançà, el Port de la Selva, Cadaqués, Roses, Castelló d’Empúries, Sant Pere Pescador, 1’Escala, Torroella de Montgrí, Pals, Begur, Palafrugell, Palamós, Calonge, CastellPlatja d'Aro, Santa Cristina d'Aro, Sant Feliu de Guíxols, Tossa de Mar, Lloret de Mar y Blanes) y a técnicos de la Junta d'Aigües de Catalunya en las que se preguntaban distintos aspectos relacionados con las formas de defensa frente a las inundaciones existentes en cada municipio y sobre aquellas que se creían necesarias llevar a cabo para conseguir una 
mayor protección. El análisis de los resultados de estas entrevistas ha dado pie a las reflexiones que se reúnen en este trabajo.

El artículo se ha dividido en cuatro apartados. En el primero se realiza una breve introducción sobre la evolución histórica de las obras hidráulicas destinadas a la protección frente a avenidas fluviales. A continuación se presenta el ámbito de estudio de la Costa Brava en relación a las características físicas (relieve, climatología e hidrología) y humanas (agricultura y turismo, principalmente) más directamente relacionadas con el origen y efectos de los desbordamientos de ríos y rieras litorales. En el tercer apartado se analizan cuáles son las obras hidráulicas existentes en estos cursos fluviales y cuáles son las previstas. Por último, se definen las líneas generales que establecen las preferencias locales en relación a medidas de adaptación frente a las inundaciones en los municipios de la Costa Brava.

\section{Evolución histórica de la obras hidráulicas para la protección frente a las inundacio-} nes fluviales

La evolución de las obras hidráulicas constata la importancia que estos dispositivos de defensa han tenido en muchas sociedades a fin de hacer frente a los efectos de las inundaciones (ver tabla 1). En las sociedades tradicionales agrarias, las obras hidráulicas de defensa podían coincidir con las infraestructuras de regadío, ya que acequias, canales de riego y otras obras se utilizaban simultáneamente para la distribución del agua y para su contención. Posteriormente, empiezan a construirse diques en los márgenes de los ríos para evitar que las aguas invadieran los núcleos de población. Muchas de estas obras, a menudo bastante precarias, resultaban fácilmente destruidas en los episodios de inundación más importantes. Estas sociedades tradicionales incluso llegaban a desviar algún curso de agua o, de forma más frecuente, eliminaban meandros con la finalidad de minimizar el riesgo de desbordamiento.

A medida que nos acercamos al s. XX, las sucesivas innovaciones tecnológicas en materia ingenieril conllevan el diseño de nuevos dispositivos hidráulicos y la utilización de nuevos materiales. Empiezan a proliferar las primeras obras de canalización en ríos y rieras, así como la construcción de embalses y otras estructuras de laminación de avenidas. En general, las sociedades tienden cada vez más a modificar el comportamiento hidrológico de los cursos fluviales con la finalidad de alejar las aguas de avenida de la población y sus bienes, sin tener demasiado en cuenta los problemas que puede generar la alteración de las condiciones naturales de los ríos y la progresiva artificialización del paisaje fluvial. Situaciones extremas se dan en muchas rieras y otros cursos menores de agua del litoral mediterráneo, donde la creciente urbanización ha motivado la construcción de multitud de obras hidráulicas para prevención de avenidas, hasta el punto que estos espacios aparecen hoy en día como áreas fuertemente estigmatizadas y abandonadas a usos marginales (Breton y Saurí, 1997). En estas zonas urbanizadas, la inundación difusa es un problema que va en constante aumento debido a que las redes de drenaje no están lo suficientemente preparadas para absorber las grandes cantidades de agua que se acumulan en estos espacios cuando las precipitaciones son intensas. Ante el aumento de este tipo de inundación los Ayuntamientos de la Costa Brava demandan soluciones estructurales para combatir esta situación.

Ya en los últimos años, y coincidiendo con un momento de reivindicación de la protección del medio ambiente y la calidad de vida, empiezan a aparecer las primeras voces 
que reclaman el establecimiento de una nueva relación entre los ríos y las sociedades a partir de la sustitución de los dispositivos hidráulicos convencionales por otros más respetuosos de los ecosistemas fluviales. Ello implica que la prevención de inundaciones tiene que ser compatible con las actuaciones encaminadas a retornar al estado más natural posible nuestros cursos fluviales. Sin embargo, a pesar de estas nuevas tendencias de cambio en la gestión de los ríos y del riesgo de inundación, todo hace suponer que la obra hidráulica convencional continuará siendo la opción mayoritariamente defendida en aquellos espacios, como la Costa Brava, en los que poco se ha actuado hasta ahora por lo que respeta al control de las fuerzas que inciden en un incremento de la vulnerabilidad, básicamente el crecimiento urbanístico.

Tabla 1

EVOLUCIÓN DE LAS OBRAS HIDRÁULICAS DE DEFENSA FRENTE A INUNDACIONES

\begin{tabular}{|l|l|}
\hline \multicolumn{1}{|c|}{ Modelo de sociedad } & \multicolumn{1}{c|}{ Obras hidráulicas } \\
\hline Sociedades tradicionales & - Acequias, canales y otras infraestructuras \\
& de regadío \\
& - Pequeñas presas \\
& - Motas y diques laterales \\
& - Desviación ocasional de algún tramo del \\
& curso fluvial \\
& - Eliminación puntual de algún meandro del \\
& curso fluvial \\
Sociedades modernas «tecnificadas» & - Canalizaciones \\
& - Embalses \\
& - Diques \\
& - Desvíos \\
& - Cubrimiento \\
& - Sistema de evacuación de aguas pluviales \\
Sociedades modernas «ambientalizadas» & - Eliminación de las obras hidráulicas «duras» \\
& - Planificación de los usos del suelo en los \\
& espacios inundables \\
\hline
\end{tabular}

FUENTE: elaboración propia.

\section{Obras hidráulicas y seguridad}

A pesar de la eficacia reiteradamente demostrada de las obras hidráulicas, también es cierto que éstas no proporcionan una protección absoluta frente al riesgo. La construcción de numerosas obras hidráulicas con la finalidad de minimizar el riesgo de inundación ha comportado que se relativice la noción de catástrofe debido al sentimiento de «falsa seguridad» que proporcionan estas obras (Calvo, 1984; Berga, 1992). La existencia de obras de defensa cada vez más sofisticadas y complejas provoca que aumenten considerablemente 
los asentamientos humanos en las zonas inundables hasta aquellos momentos respetadas y que se inicie un círculo vicioso donde cada vez se precisen protecciones más grandes y, en consecuencia, los asentamientos humanos serán cada vez más importantes (Parker, 1995) En otras palabras, podemos decir que la vulnerabilidad al riesgo crece, en muchos espacios, más rápidamente que la adaptación al mismo.

La confianza o la percepción optimista que la sociedad ha depositado en los embalses de laminación, las canalizaciones, etc., no se corresponde siempre con la realidad, ya que su diseño y construcción parten de una conceptualización relativa del riesgo: por una parte, porque la reducción absoluta del riesgo no existe y, por otra, porque el nivel de riesgo aceptable que pueden ofrecer las obras hidráulicas ha de compararse con los beneficios que comporta este nivel de riesgo para la sociedad que habita en el espacio potencialmente afectado. Así, en última instancia, el nivel de protección que proporcionan las obras hidráulicas actuales es el resultado de la optimización básicamente económica (balance entre costos y beneficios) del territorio expuesto a los efectos de una inundación, teniendo en cuenta que nunca se podrá eliminar la posibilidad de padecer una catástrofe desde el momento que puede tener lugar un acontecimiento que sobrepase la capacidad de contención de la obra hidráulica y producirse un desastre incluso de mayores consecuencias (Saurí, 1991). El derrumbe de un embalse, por ejemplo, puede provocar una catástrofe seguramente más grave que una avenida de período de retorno muy elevado. Las nuevas tecnologías en ingeniería hidráulica hacen que la posibilidad de rotura de un embalse sea muy baja, pero no debe olvidarse que siempre existe un riesgo residual, como lo demuestran algunos episodios recientes, entre los cuales el desastre de Tous de 1982 es seguramente el más conocido. También pudimos comprobar como en la catástrofe de Biescas, acaecida el verano de 1996, el agua superó algunos de los diques transversales que se habían construido en el barranco de Arás y que teóricamente estaban preparados para contener caudales importantes y proteger, así, la zona del cámping. A pesar de ello y por diversos motivos (financiación pública de las obras, no necesidad de modificar el comportamiento humano, etc.) las obras hidráulicas se mencionan continuamente como las mejores alternativas para afrontar el riesgo de inundación.

\section{La Costa Brava y las inundaciones}

El espacio definido como Costa Brava comprende básicamente la fachada litoral de las comarcas gerundenses, limitando al norte con la frontera francesa y al sur con el río Tordera (ver mapa 1).

La red hidrográfica de la Costa Brava está constituida básicamente por tres ríos (Muga, Fluvià y Ter) y multitud de rieras y torrentes (ver tabla 2).

En el caso de los tres ríos más importantes (el Fluvià, el Ter y la Muga), no presentan un régimen torrencial, pero durante los episodios de inundación sus aguas acostumbran a ocupar una amplia superficie. Además, en el caso de la Muga y el Ter, existen a lo largo de su recorrido diversos embalses que contribuyen en buena medida a laminar las puntas de avenida. A excepción de estos tres ríos, el resto de cursos fluviales de la Costa Brava son de recorrido generalmente corto y de régimen torrencial, por lo que pueden concentrar importantes caudales de agua en poco espacio de tiempo.

Así pues, las condiciones naturales de la Costa Brava, principalmente el relieve (pendiente), las precipitaciones (de fuerte intensidad y corta duración) y la compleja red hidrográfica, son los factores físicos desencadenantes de las importantes inundaciones que 
Tabla 2

PRINCIPALES RÍOS Y RIERAS DE LA COSTA BRAVA Y CAUDALES MÁXIMOS ESTIMADOS

\begin{tabular}{|l|c|}
\hline Curso fluvial & Caudal máximo estimado $\left(\mathbf{m}^{3} \mathbf{s}\right)$ \\
\hline Río Muga & 1.900 (en Castelló d’Empúries) \\
Río Fluvià & 2.000 (en Garrigàs) \\
Río Ter & 2.400 (en Torroella de Montgrí) \\
Riera de Portbou & 140 \\
Riera de Molinars & 210 \\
Riera de Valleta & 320 \\
Riera de Rubies & 230 \\
Rieras de Coana, Ginjolers, Forquilla y Trencada & - \\
Riera de Tamariu & 60 \\
Riera de Llafranc & 50 \\
Riera Aubi & 330 \\
Riera de Calonge & 500 \\
Riera de Ridaura & 600 \\
Riera de Sant Pol & 50 \\
Rieras de Tueda, Sant Amancio i les Comes & - \\
Riera de Tossa & 120 \\
Riera de Fanals & - \\
Riera de Lloret & 220 \\
Riera de Blanes & 100 \\
\hline
\end{tabular}

FUENTE: elaboración propia a partir de Generalitat de Catalunya (1993) y López Bustos (1980).

padece este espacio. Pero han sido las actividades turísticas y los equipamientos que a ellas van asociadas, el incremento demográfico (permanente o temporal) y la construcción de diversas infraestructuras, los factores responsables del aumento de la vulnerabilidad a las inundaciones que experimenta la Costa Brava. La gran urbanización que ha experimentado la Costa Brava (y el litoral mediterráneo en general) ha comportado que el medio construido haya ocupado espacios de riesgo (antiguas marismas, llanuras de inundación de los ríos, etc.), aumentando, consecuentemente, la susceptibilidad de padecer los efectos de las inundaciones. Algunos ejemplos claros son las urbanizaciones Mas Pinell (situada en la desembocadura del río Ter) y Mas Fumats (Roses) y los cámpings Valldaro y Riembau (tramo bajo del río Ridaura), siendo el caso más espectacular la urbanización Empúriabrava, en el municipio de Castelló d’Empúries, que se extiende por el margen izquierdo del río Muga y ocupa una amplia superficie de antiguas marismas.

A pesar de los importantes daños que actualmente causan las inundaciones en la Costa Brava y que afectan a sectores tan diversos como la agricultura, los núcleos de población, los equipamientos turísticos y la infraestructura viaria, principalmente, también se constata que en los últimos años los espacios y actividades más afectados han sido los relacionados con el sector turístico (urbanizaciones, cámpings, hoteles, etc.). Y 
no sólo debido al aumento de la ocupación humana en espacios susceptibles de ser afectados por las aguas sino también porque esta ocupación humana ha modificado los parámetros responsables de la formación de caudales extraordinarios, especialmente aquellos que inciden en la retención de la escorrentía superficial por medios naturales. Es por todo ello que la construcción de numerosas obras hidráulicas en ríos y rieras ha sido (y sin duda alguna continúa siendo) la forma de adaptación preferida por estas sociedades.

\section{Estado actual de las obras hidráulicas en los ríos y rieras de la Costa Brava}

En la tabla 3 se resume el tipo y las características de las obras de infraestructura hidráulica que predominan en la Costa Brava.

Tabla 3

OBRAS DE INFRAESTRUCTURA HIDRAUULICA EXISTENTES EN LOS RIOS Y RIERAS DE LOS MUNICIPIOS DE LA COSTA BRAVA

\begin{tabular}{|c|c|c|c|c|c|c|c|c|}
\hline \multirow[b]{2}{*}{ Municipio } & \multicolumn{8}{|c|}{ Obras hidráulicas } \\
\hline & 1 & 2 & 3 & 4 & 5 & 6 & 7 & 8 \\
\hline Portbou & $\mathrm{x}$ & & & & & & & - \\
\hline Colera & $\mathrm{x}$ & & $\mathrm{x}$ & & & & & $\mathrm{P}$ \\
\hline Llançà & & & & & & & & $\mathrm{P}$ \\
\hline el Port de la Selva & & & & & & & & - \\
\hline Cadaqués & $\mathrm{x}$ & & & & & & & $\mathrm{P}$ \\
\hline Roses & & & $\mathrm{x}$ & & & $\mathrm{x}$ & $\mathrm{x}$ & - \\
\hline Castelló d’Empúries & & $\mathrm{x}$ & & & & & & $\mathrm{P}$ \\
\hline Sant Pere Pescador & $\mathrm{x}$ & & & & & & & - \\
\hline l’Escala & $\mathrm{x}$ & & & & & & & - \\
\hline Torroella de Montgrí & & & $\mathrm{x}$ & & & & & $\mathrm{P}$ \\
\hline Pals & & & $\mathrm{x}$ & & & & & $\mathrm{T}$ \\
\hline Begur & $\mathrm{x}$ & & & & & & & - \\
\hline Palafrugell & $\mathrm{x}$ & & $\mathrm{x}$ & & & & & $\mathrm{P}$ \\
\hline Palamós & & & $\mathrm{x}$ & $\mathrm{x}$ & & & & - \\
\hline Calonge & & & & $\mathrm{x}$ & & & & $\mathrm{P}$ \\
\hline Castell-Platja d'Aro & $\mathrm{x}$ & $\mathrm{x}$ & & & & & $\mathrm{x}$ & $\mathrm{T}$ \\
\hline Santa Cristina d'Aro & & & & & $\mathrm{x}$ & & & $\mathrm{P}$ \\
\hline Sant Feliu de Guíxols & $\mathrm{x}$ & & & & & & $\mathrm{x}$ & $\mathrm{T}$ \\
\hline Tossa de mar & & & $\mathrm{x}$ & & & & & $\mathrm{T}$ \\
\hline Lloret de Mar & $\mathrm{x}$ & & $\mathrm{x}$ & & & & $\mathrm{x}$ & $\mathrm{P}$ \\
\hline Blanes & $\mathrm{x}$ & & $\mathrm{x}$ & $\mathrm{x}$ & & & & $\mathrm{P}$ \\
\hline
\end{tabular}

1 Canalización, 2 Mota, 3 Muro de contención, 4 Desvío, 5 Embalse de laminación, 6. Trampa de retención de sedimentos, 7. Cubrimiento, 8 Sistema separativo de aguas pluviales $(\mathrm{T}=$ total; $\mathrm{P}=$ parcial) 
Como puede contrastarse en la tabla 3, en todos los municipios (a excepción de Llançà y el Port de la Selva) existe algún tipo de obra hidráulica en el río o riera que cruza estos municipios, que van desde las obras de ingeniería hidráulica «tradicional» (motas, diques, etc.) hasta aquellas más modernas (canalizaciones, embalses, sistema de evacuación aguas pluviales, etc.). Las obras hidráulicas constituyen la medida de adaptación a las inundaciones preferida por las administraciones locales en detrimento de otras medidas de carácter no-estructural. La justificación a esta elección es fácilmente imaginable: la intensa urbanización de los espacios inundables hace inviable pensar en aplicar otras medidas, como la planificación de los usos de la llanura de inundación, respetando los terrenos próximos a los cursos fluviales para usos alternativos a la construcción, por el sencillo hecho que estos espacios inundables ya se encuentran ocupados. Ante esta situación, expresada por muchas de las personas entrevistadas, solo queda una solución: la obra hidráulica. Sin embargo, en muchos casos constatamos como actualmente se sigue edificando aguas arriba de las rieras, siendo el paso siguiente la canalización.

Pero ello no siempre ha sido así. Las sociedades que venimos llamando «tradicionales» guardaban más respeto a las fuerzas de la naturaleza que nuestras sociedades más modernas, seguramente porque no disponían de la tecnología necesaria para dominar estas fuerzas. Tanto es así que la localización de los núcleos de población tenía una posición elevada en relación a los ríos y rieras, si bien actualmente muchos de ellos han prácticamente integrado algunas rieras en el trazado urbano (Castell-Platja d'Aro, Llançà, el Port de la Selva, 1’Escala, por ejemplo).

\section{Gestión municipal y obras hidráulicas}

En este apartado se analizan los resultados obtenidos de las entrevistas realizadas a diferentes responsables municipales de los Ayuntamientos de la Costa Brava. El método utilizado ha sido la entrevista en profundidad. Se han tratado aspectos muy diversos sobre la problemática de las inundaciones: el impacto de las inundaciones en el término municipal, las características físicas de los episodios de inundación, la caracterización de la vulnerabilidad territorial, económica y social a estos fenómenos y, finalmente, las formas de adaptación desarrolladas hasta el momento para hacer frente al riesgo. Si bien esta metodología presenta algunos inconvenientes (por ejemplo, en algunos aspectos, visión subjetiva de la persona entrevistada), el resultado fue satisfactorio en el sentido que en líneas generales las opiniones recogidas expresan la opinión mayoritaria que hay sobre esta temática desde el punto de vista de la Administración Local.

Cuando se pregunta a los responsables municipales que medidas de defensa serían necesarias para minimizar el riesgo potencial de inundación, la amplia mayoría coinciden en reivindicar la mejora de la infraestructura hidráulica existente y la necesidad de construir nuevos dispositivos hidráulicos de defensa (ver tabla 5). 
Tabla 5

MEDIDAS DE ADAPTACIÓN MÁS EFICACES FRENTE A LAS INUNDACIONES SEGÚN LOS RESPONSABLES MUNICIPALES DE LA COSTA BRAVA*

\begin{tabular}{|c|c|}
\hline Municipio & Formas de adaptación más eficaces \\
\hline Portbou & $\begin{array}{l}\text { Obras hidráulicas } \\
\text { Sistema de evacuación de aguas pluviales } \\
\text { Revegetación y estabilización de taludes }\end{array}$ \\
\hline Colera & $\begin{array}{l}\text { Obras hidráulicas } \\
\text { Ampliación del sistema de evacuación de aguas pluviales } \\
\text { Revegetación y estabilización de taludes }\end{array}$ \\
\hline Llançà & $\begin{array}{l}\text { Obras hidráulicas } \\
\text { Ampliación del sistema de evacuación de aguas pluviales } \\
\text { Planes de Emergencia }\end{array}$ \\
\hline el Port de la Selva & $\begin{array}{l}\text { Obras hidráulicas } \\
\text { Contratación de Seguros } \\
\text { Revegetación y estabilización de taludes }\end{array}$ \\
\hline Cadaqués & Obras hidráulicas \\
\hline Roses & $\begin{array}{l}\text { Obras hidráulicas } \\
\text { Ampliación del sistema de evacuación de aguas pluviales } \\
\text { Planes de emergencia }\end{array}$ \\
\hline Castelló d’Empúries & Ampliación del sistema de evacuación de aguas pluviales \\
\hline Sant Pere Pescador & Mejora de las infraestructuras viarias \\
\hline 1’Escala & $\begin{array}{l}\text { Obras hidráulicas } \\
\text { Regulación urbanística de áreas inundables }\end{array}$ \\
\hline Torroella de Montgrí & $\begin{array}{l}\text { Obras hidráulicas } \\
\text { Ayudas públicas postcatástrofe }\end{array}$ \\
\hline Pals & $\begin{array}{l}\text { Ampliación del sistema de evacuación de aguas pluviales } \\
\text { Planes de Emergencia } \\
\text { Contratación de seguros }\end{array}$ \\
\hline Begur & $\begin{array}{l}\text { Mejora del lecho de las rieras } \\
\text { Planes de emergencia }\end{array}$ \\
\hline Palafrugell & $\begin{array}{l}\text { Ampliación del sistema de evacuación de aguas pluviales } \\
\text { Obras hidráulicas } \\
\text { Ayudas públicas postcatástrofe }\end{array}$ \\
\hline Palamós & $\begin{array}{l}\text { Ampliación del sistema de evacuación de aguas pluviales } \\
\text { Obras hidráulicas }\end{array}$ \\
\hline
\end{tabular}




\begin{tabular}{|l|l|}
\hline Municipio & Formas de adaptación más eficaces \\
\hline Calonge & $\begin{array}{l}\text { Obras hidráulicas } \\
\text { Ampliación del sistema de evacuación de aguas pluviales } \\
\text { Ayudas públicas postcatástrofe }\end{array}$ \\
Castell-Platja d’Aro & $\begin{array}{l}\text { Obras hidráulicas } \\
\text { Ampliación del sistema de evacuación de aguas pluviales }\end{array}$ \\
Santa Cristina d'Aro & $\begin{array}{l}\text { Obras hidráulicas } \\
\text { Ampliación del sistema de evacuación de aguas pluviales } \\
\text { Ayudas públicas postcatástrofe }\end{array}$ \\
Sant Feliu de Guíxols & $\begin{array}{l}\text { Obras hidráulicas } \\
\text { Tossa de Mar }\end{array}$ \\
Lloret de Mar & $\begin{array}{l}\text { No hay problemas importantes de inundaciones } \\
\text { Obras hidráulicas } \\
\text { Regulación urbanística de las zonas inundables } \\
\text { Ampliación del sistema de evacuación de aguas pluviales }\end{array}$ \\
& $\begin{array}{l}\text { Obras hidráulicas } \\
\text { Ampliación del sistema de evacuación de aguas pluviales }\end{array}$ \\
\hline
\end{tabular}

* Las medidas de adaptación que se consideran más eficaces se relacionan por orden de preferencia.

FUENTE: Entrevistas realizadas a los Ayuntamientos de la Costa Brava 1995/1996.

Del total de 21 municipios entrevistados, 14 de ellos (es decir, el 66,6 \% del total) opinan que la medida de adaptación más eficaz para minimizar el riesgo de inundación en su municipio son las obras hidráulicas en los ríos y rieras. A la vez, significativamente, del resto de municipios que optaron por otra medida como la más óptima, Palafrugell y Palamós enumeran las obras hidráulicas en segundo lugar. Es igualmente interesante hacer notar que es de opinión común entre todas las personas entrevistadas la necesidad urgente de construir obras hidráulicas, hasta el punto de afirmar en muchos casos que una vez se dispongan de las obras reclamadas se habrán terminado para siempre las inundaciones y los problemas que de ellas se derivan.

Ello nos lleva a pensar que, a pesar de que actualmente desde otros organismos se empieza a percibir un cambio de tendencia en relación a la construcción de obras hidráulicas en los cursos fluviales como medida más adecuada para hacer frente a las avenidas, a nivel local continúa imperando la defensa de la construcción de dispositivos hidráulicos como única solución contemplada. Las resistencias sociales, especialmente a escala local, pueden llegar a ser importantes y ciertamente legítimas, más cuando muchas veces la urbanización sin planeamiento previo hace inviable la opción de la regulación de los usos del suelo, siendo la modificación del elemento natural la única alternativa posible.

En concreto, entre las peticiones municipales de mejora o construcción de obras hidráulicas destacan aquellas que reivindican la construcción de obras de canalización de los cursos fluviales (a fin de incrementar al máximo la sección del lecho fluvial y conseguir así una rápida evacuación de las aguas), especialmente cuando éstos circulan por alguna zona urbana. 
Otra de las obras hidráulicas más ampliamente reivindicadas son los embalses de laminación en la cabecera de algunas rieras. Este dispositivo hidráulico es solicitado básicamente por aquellos municipios de topografía abrupta, como son Colera, Llançà, el Port de la Selva y Roses. Las fuertes pendientes de estas zonas, debidas a la presencia de la Serra de Roda concentran en pocos instantes caudales importantes de agua de difícil control a su paso por estas poblaciones, aunque muchas de estas rieras estén ya canalizadas. También por aquellos municipios que ya no pueden mejorar su canalización, como es el caso de Tossa de Mar.

Tabla 6

OBRAS HIDRÁULICAS DE PREVENCIÓN DE INUNDACIONES RECLAMADAS DESDE LOS AYUNTAMIENTOS DE LA COSTA BRAVA

\begin{tabular}{|c|c|}
\hline Municipio & Obras hidráulicas reclamadas \\
\hline Portbou & $\begin{array}{l}\text { Mejora del sistema de evacuación de aguas } \\
\text { Modificar el lecho de la riera } \\
\text { Canalización de la riera }\end{array}$ \\
\hline Colera & $\begin{array}{l}\text { Construcción de muros de hormigón en la riera } \\
\text { Embalses de cabecera } \\
\text { Sistema de evacuación de aguas separativa }\end{array}$ \\
\hline Llançà & $\begin{array}{l}\text { Canalización de la riera Valleta (ya proyectada) } \\
\text { Embalse de cabecera (proyectado pero cuestionado desde } \\
\text { Junta d'Aigües por su elevado coste) }\end{array}$ \\
\hline el Port de la Selva & $\begin{array}{l}\text { Embalse de cabecera } \\
\text { Canalización de la riera }\end{array}$ \\
\hline Cadaqués & $\begin{array}{l}\text { Mejora de la canalización de la riera } \\
\text { Sistema de evacuación de aguas separativa }\end{array}$ \\
\hline Roses & $\begin{array}{l}\text { Instalaciones de bombeo de aguas (proyecto) } \\
\text { Cubrimiento de la riera Trencada }\end{array}$ \\
\hline Castelló d’Empúries & Limpieza periódica del lecho del río Muga y otros cursos de agua \\
\hline Sant Pere Pescador & Mejora del sistema de evacuación de aguas residuales urbanas \\
\hline 1’Escala & $\begin{array}{l}\text { Canalización de la riera Montgó } \\
\text { Sistema de evacuación de aguas pluviales en Riells } \\
\text { Dique de contención de aguas pluviales en la montaña del Montgrí }\end{array}$ \\
\hline Torroella de Montgrí & $\begin{array}{l}\text { Canalización de los últimos tramos de algunos canales } \\
\text { Estación de bombeo en el torrente d'en Planes }\end{array}$ \\
\hline Pals & - \\
\hline Begur & Mejora de la canalización de la riera de Esclanyà \\
\hline
\end{tabular}




\begin{tabular}{|l|l|}
\hline Municipio & Obras hidráulicas reclamadas \\
\hline Palafrugell & $\begin{array}{l}\text { Canalización de la riera Aubi (proyecto) } \\
\text { Construcción del sistema de evacuación de aguas separativa } \\
\text { Mejora del sistema de evacuación de aguas }\end{array}$ \\
Calonge & $\begin{array}{l}\text { Mejora del sistema de evacuación de aguas } \\
\text { Canalización de la riera Ridaura (proyecto) } \\
\text { Construcción del sistema de evacuación de aguas pluviales en } \\
\text { algunas urbanizaciones donde falta } \\
\text { Construir un espigón que facilite la salida de las aguas de la } \\
\text { rieras al mar } \\
\text { Santa Cristina d'Aro } \\
\text { Mejora, en general, de las rieras, especialmente en la zona del } \\
\text { campo de golf } \\
\text { Mejora del sistema de evacuación de aguas pluviales, } \\
\text { especialmente en la zona del campo de golf } \\
\text { Canalización de la riera de Vilartagues }\end{array}$ \\
Llossa de Mar & $\begin{array}{l}\text { Mejora de las conducciones del sistema de evacuación de aguas } \\
\text { Cubrimento y canalización de la riera, en el tramo de la zona } \\
\text { deportiva (proyecto) } \\
\text { Cubrimento de un tramo de la riera de Fanals (proyecto) } \\
\text { Mejora de las obras hidráulicas existentes }\end{array}$ \\
\hline
\end{tabular}

FUENTE: Entrevistas realizadas a los Ayuntamientos de la Costa Brava 1995/1996.

Por último cabe mencionar que entre las obras de infraestructura hidráulica más reivindicadas destacan especialmente las referentes a la mejora del sistema de evacuación de aguas pluviales (13 municipios la consideran una medida necesaria). La intensa urbanización de la Costa Brava requiere de un buen sistema de evacuación de aguas pluviales que evite las acumulaciones de agua pero que falta en muchos municipios. De hecho, como ya se ha comentado anteriormente, la inundación difusa es un tipo de riesgo más reciente, a la que los municipios están todavía poco adaptados. Además, al contrario que otras obras, los costes en este caso han de asumirse mayoritariamente desde las administraciones locales.

\section{Conclusiones}

La Costa Brava es actualmente uno de los espacios de Cataluña más afectados por las inundaciones provocadas por sus ríos y rieras. Las especiales características topográficas y climatológicas de este espacio, pero especialmente un comportamiento humano poco atento a las particularidades del medio fluvial, son los responsables directos del importante incremento de la vulnerabilidad experimentado en las últimas décadas por este espacio en relación a estos fenómenos extremos de la naturaleza. 
Mapa 1

Localización de los municipios de la Costa Brava.

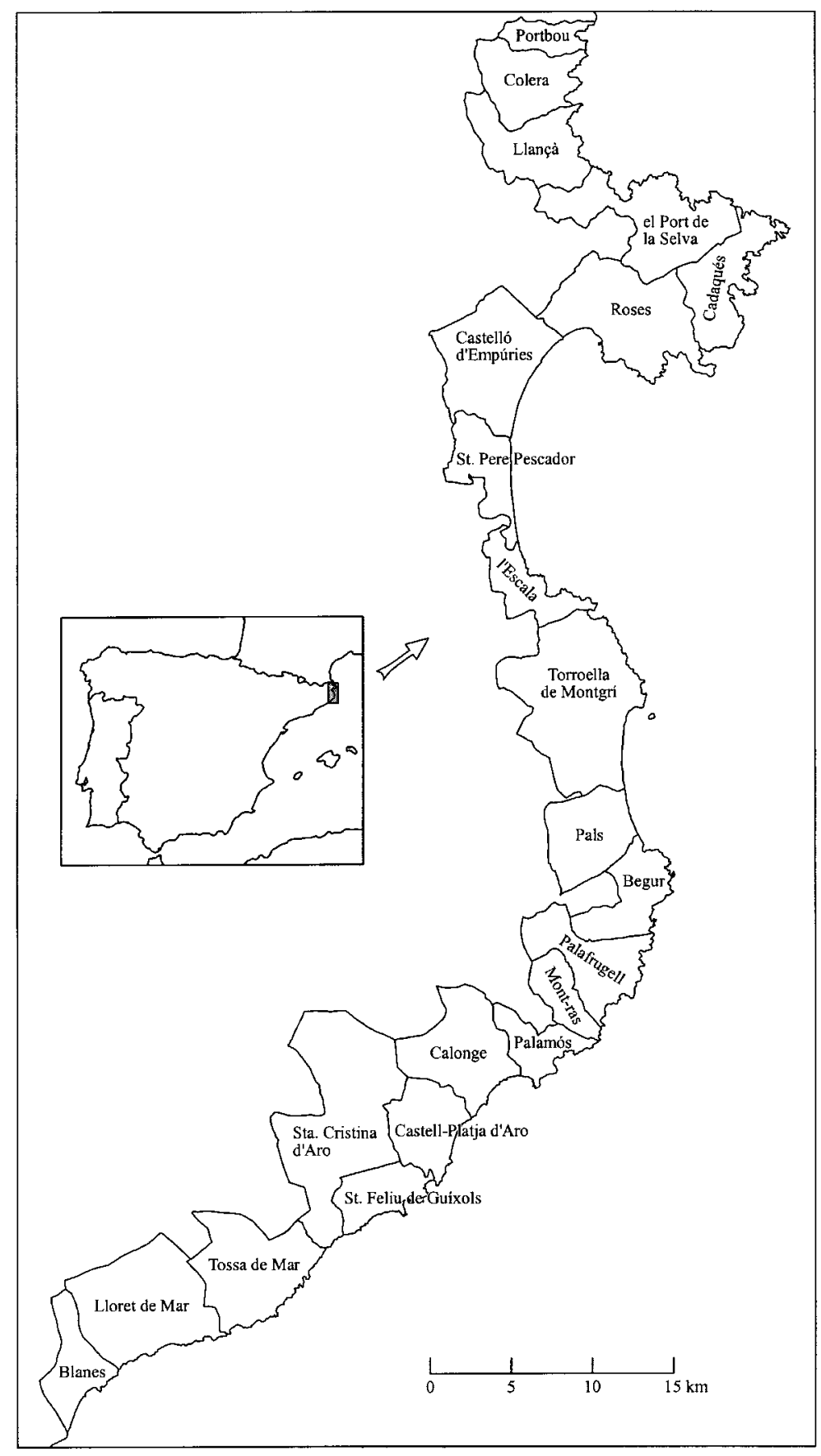


Las inundaciones que tuvieron lugar en octubre de 1994 demostraron que a pesar de las muchas obras hidráulicas de prevención de inundaciones existentes, los daños no habían hecho sino que aumentar. Roses, Llançà, el Port de la Selva, l'Escala, Palamós, Calonge, Cadaqués, Sant Pere Pescador y 1’Estartit (Torroella de Montgrí) sufrieron cuantiosas pérdidas económicas debidas al desbordamiento de ríos y rieras, agravados en el caso de 1'Estartit por importantes deslizamientos de tierras que afectaron residencias y carreteras (Saurí y otros, 1993).

Del conjunto de formas de adaptación al riesgo de inundación existentes, las obras hidráulicas en ríos y rieras han sido y son todavía hoy la opción preferida por parte de la población de la Costa Brava. De hecho, esta preferencia no es exclusiva de este ámbito sino común a muchos espacios amenazados por este tipo de fenómenos. La progresiva urbanización del litoral gerundense provoca el constreñimiento de ríos y rieras dentro de las zonas urbanas, acentuando el riesgo en los momentos de crecida, pero también causando un importante impacto ambiental cada vez más valorado por nuestra sociedad.

En definitiva, en la Costa Brava, como en tantos otros espacios del litoral español, el círculo vicioso «inundación-obra hidráulica-inundación» puede seguir determinando la vulnerabilidad de estas áreas al riesgo de inundación. Los gestores municipales deberían hacer un esfuerzo por controlar las fuerzas inductoras que inciden directamente en el incremento de la vulnerabilidad al riesgo. Este planteamiento debería basarse en las siguientes cuestiones básicas: 1) estas obras hidráulicas no garantizan una protección absoluta, a pesar de los importantes costes económicos, sociales y medioambientales que comportan, 2) el importante crecimiento urbanístico en los espacios tradicionalmente inundables seguramente provoca que las medidas de adaptación actuales, construidas hace ya algunos años, no sean del todo seguras, 3 ) se hace necesario valorar la alternativa de emprender medidas de carácter no-estructural para hacer frente a las avenidas, y 4) el riesgo cero es imposible de conseguir. Una posible solución a esta problemática podría basarse en dejar de inculcar a la población conceptos de peligro y potenciar estrategias futuras de gestión del riesgo basadas en la conservación del patrimonio natural (marismas, parques fluviales, etc.), usos que de alguna forma se benefician de las inundaciones y los desbordamientos periódicos. En otras palabras, se regula la ocupación humana de un espacio no tanto por el peligro que comporta como por sus valores ambientales. Evidentemente, esta sería una opción difícil de implementar en aquellos espacios inundables ya ocupados y transformados por la acción humana, pero sí a tener en cuenta en aquellos que se encuentran amenazados por el crecimiento urbanístico que caracteriza a este territorio.

\section{Bibliografía}

BERGA CASAFONT, L. (1992): «Avenidas fluviales con afecciones urbanas», en Dolz, J.; Gómez, M.; Martín, J.P. (eds.): Inundaciones y redes de drenaje urbano, Barcelona, Universitat Politècnica de Catalunya, Escuela T. S. de Ingenieros de Caminos, Canales y Puertos.

BRETON, F.; SAURÍ, D. (1997): «Ephemeral streams and urban growth in coastal areas: the case of Catalonia, Spain», comunicación presentada a EUCC Coastline, Nápoles (en prensa).

CALVO GARCÍA-TORNEL, F. (1984): «La geografía de los riesgos», Geocrítica, 54.

GENERALITAT DE CATALUNYA (1983): Pla d'obres $i$ correccions a fer a la Xarxa

Hidrològica de Catalunya. Obres de defensa i correcció i proposta de previsió d'avin- 
gudes, Barcelona, Generalitat de Catalunya, Departament de Política Territorial i Obres Públiques, Departament d'Agricultura, Ramaderia i Pesca.

GENERALITAT DE CATALUNYA (1994a) Pla hidrològic de les conques internes de Catalunya. Projecte, Barcelona, Generalitat de Catalunya, Departament de Política Territorial i Obres Públiques, Departament de Medi Ambient.

GENERALITAT DE CATALUNYA (1994b): Atles urbanistic de la Costa Brava de Catalunya, Barcelona, Generalitat de Catalunya, Departament de Política Territorial i Obres Públiques, Direcció General d'Urbanisme, Institut Cartogràfic de Catalunya.

GIL OLCINA, A.; MORALES GIL, A. (DE.) (1989): Avenidas fluviales e inundaciones en la cuenca del Mediterráneo, Alicante, Instituto Universitario de Geografía.

LÓPEZ BUSTOS, A. (1980): «Antecedentes para una historia de las avenidas en los ríos del Pirineo Oriental», Revista de Obras Públicas, mayo, pp. 369-383.

PARKER, D.J. (1995): «Floodplain development policy in England and Wales», Applied Geography, vol. 15, 4, pp. 341-363.

PARKER, D.J. (1995): «Floodplain development policy in England and Wales», Applied Geography, vol. 15, 4, pp. 341-363.

SAURÍ, D. (1991): «Vulnerabilitat i adaptació al risc d'avinguda: l'aiguat de 1982 al Pirineu Català», ponencia presentada al 10è Curs d'Estiu d'Estudis Pirinencs, La Seu d’Urgell.

SAURÍ PUJOL, D.; RIBAS, A.; SORRIBAS, E.; ROSET, D. (1993): Inundacions i societat al Baix Ter, Girona, Dalmau Carles Pla.

SAURÍ PUJOL, DAVID (1997): «Les inundacions», Quaderns d'Ecologia Aplicada, 14 Barcelona, Diputació de Barcelona. 